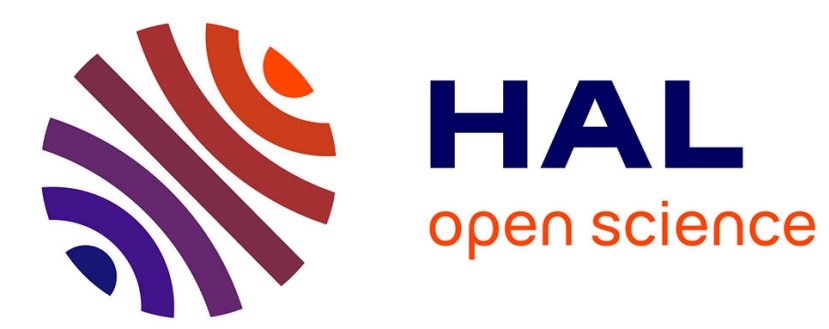

\title{
Poétique de l'Arcadie, de Virgile à Bonnefoy
}

Franck Collin

\section{- To cite this version:}

Franck Collin. Poétique de l'Arcadie, de Virgile à Bonnefoy. Bulletin de l'association Guillaume Budé , 2006, 10.3406/bude . hal-02344178

\section{HAL Id: hal-02344178 https://hal.science/hal-02344178}

Submitted on 4 Nov 2019

HAL is a multi-disciplinary open access archive for the deposit and dissemination of scientific research documents, whether they are published or not. The documents may come from teaching and research institutions in France or abroad, or from public or private research centers.
L'archive ouverte pluridisciplinaire HAL, est destinée au dépôt et à la diffusion de documents scientifiques de niveau recherche, publiés ou non, émanant des établissements d'enseignement et de recherche français ou étrangers, des laboratoires publics ou privés. 


\section{Poétique de l'Arcadie, de Virgile à Bonnefoy}

Communication prononcée à la journée d'étude L'Antiquité est-elle moderne ? organisée par Émilia Ndiaye et Alain Malissard à l'Université d'Orléans, le 06 avril 2006. Publiée dans le Bulletin de l'Association Guillaume Budé, n², p. 92-122, 2006.

On semble dénier à la poésie, aujourd'hui, qu'elle puisse prétendre, par-delà un jeu formel sur la langue, à une véritable quête de sens. Dans le cas de cette dernière hypothèse, l'élection d'un lieu géographique est déterminante, parce que seul le lieu fonde cette expérience du sens, qui suscite à la fois l'immédiateté du vécu et sa transcendance dans le proche. Mais rien n'est en même temps aussi insituable, et difficile à définir que ce lieu. J'en prendrai pour exemple l'Arcadie de Virgile, qui constitue une poétique matricielle et mouvante chez notre poète latin, au même titre que ce qu'Yves Bonnefoy nommerait, en sa langue, un « arrièrepays $»^{1}$. Mais loin de confondre les démarches de ces deux poètes que deux mille ans séparent, je voudrais montrer que leur poétique, dans leurs spécificités, vise la construction de cette conscience du sens poétique.

Héritière d'un fort substrat mythique, l'Arcadie est devenue, avec Virgile, le paradigme de toute patrie poétique. Cette métamorphose, qui l'a fait entrer dans la littérature universelle, dépasse les facteurs idéologiques de son époque qui reconnaissait les Arcadiens comme les représentants d'une protohumanité, et les inventeurs des arts premiers porteurs de la civilisation. L'originalité de Virgile est d'avoir considéré la poésie, dès les Bucoliques, comme le premier de ces arts, capable de façonner l'homme, et nul doute qu'il n'ait cru en cette finalité et ne l'ait reprise comme une formule d'espérance au moment où agonisait la République romaine.

\footnotetext{
${ }^{1}$ Bonnefoy, Yves, L'Arrière-pays, 1972, Skira, Champs Flammarion.
} 
Les Bucoliques ne sont donc pas, à nos yeux, la simple transposition de l'idylle théocritéenne en Arcadie $^{2}$, qui véhiculerait le stéréotype mièvre et kitsch d'un monde pastoral à l'innocence retrouvée. Il s'agit bien plus, avec ce premier recueil, de l'élaboration d'une poétique de l'Arcadie, dans un sens double : celui, certes, des moyens d'écriture mis en œuvre (stylistique et métrique), mais encore celui d'une philosophie esthétique (métapoétique). La lecture de Bonnefoy ne peut, à sa manière, critique et créatrice, que corroborer la réalité de cette poétique, qui, loin de se limiter aux Bucoliques, trouvent précisément sa cohérence dans les deux autres recueils de Virgile.

Il ne s'agira donc pas de retrouver, chez Virgile, l'Arcadie réelle de Grèce, mais une Arcadie recréée, un territoire rêvé, que je m'attacherai à définir selon trois axes : celui, d'abord, de l'Arcadie lyrique, terre du chant et de la Parole originaire ; puis celui de l'Arcadie terrienne, dans son lien à la nature et au monde ; celui, enfin, de l'Arcadie métaphysique, qui donne un visage aux mystères ontologiques qui occupent la condition humaine.

\section{LE LYRISME ARCADIEN COMME PANODIE}

L'imitation que Virgile fait de Théocrite dans les Bucoliques ne doit pas occulter une originalité profonde du poète latin. L'intention de Virgile est de remonter à un état antérieur de la Parole poétique. L'Arcadie représente le mieux cet état premier parce que, dans la conscience latine, les Arcadiens sont aux origines les plus reculées de l'homme. C'est une dimension manifestement absente des Idylles.

Le mythe rapporte que l'Arcadie fut la terre de l'invention du lyrisme. Invention qui fut double. La première fois, ce fut Hermès, le dieu du Cyllène, qui inventa la lyre, avec la coquille d'une tortue, mais la musique l'intéressant peu (Hermès est un dieu « commerçant »), il remit l'instrument à Apollon en échange de bœufs ${ }^{3}$, et fit donc que la destinée de la lyre devienne entièrement apollinienne. La seconde fois, c'est Pan - dieu du Lycée, et fils d'Hermès selon les généalogies - qui inventa la syrinx et se l'appropria :

\footnotetext{
${ }^{2}$ Thèse régulièrement admise depuis PANOFSKY, Erwin : L'Oeuvre d'art et ses significations, Gallimard, p.282.

${ }^{3}$ PSEUDO-HOMERE, Hymne homérique à Hermès.
} 
instituit $^{4}$

(2) Panaque, qui primus calamos non passus inertis 5 .

Les circonstances de cette invention importent : c'est en voyant la nymphe Syrinx se soustraire à ses avances, que Pan rassembla quelques « chalumeaux » (calamos) et leur confia de plaintifs soupirs qui se changèrent en musique. Pan exprime son ressenti dans l'état premier et brut des forces vitales qui l'assiègent, et qu'il libère, sans filtre, au moyen de ce nouvel instrument, et non à travers des mots articulés. Il se fait donc la voix de la nature, la bouche de l'Être au moyen de sons modulés qui sont plus que la signifiance des mots.

Il en va tout autrement de l'art d'Apollon qui élabore une poétique épurée de la mesure, et qui ne restitue que partiellement les forces brutes de la vie, après les avoir mises à distance

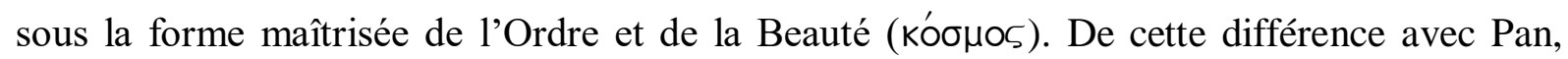
Ovide rend compte, quand il représente le concours amébée qui opposa Pan à Apollon, avec le Tmolus pour juge ${ }^{6}$.

Pour Ovide, l'art panique est déclassé par rapport à l'art apollinien car la technique, chez ce dernier, est plus consommée, plus «artificielle » certes, mais maîtrisant mieux ses effets. Le résultat en est cette «douceur» séductrice (captus) qu'Apollon déverse de son instrument, avec un talent d'expert. A côté de lui, l'art de Pan, demeure sauvage et mal dégrossi, le dieu étant un habitant des grottes et des montagnes ${ }^{7}$ qui ne connaît que des accents rudes et « barbares », c'est-à-dire étrangers à l'image polie qu'une certaine doxa se forge de l'homme ${ }^{8}$.

Virgile n'oppose certes pas à ce point les deux arts poétiques. Il retient Apollon en qualité de vo $\mu \varepsilon v \varsigma^{9}$, et le cite même plus fréquemment que $\operatorname{Pan}^{10}$. L'apanage du dieu étant la beauté plastique (formosum), tout berger-poète tente d'atteindre cette beauté de la forme (forma) qui compensera leur disgrâce physique ou leur mode de vie par trop humble ${ }^{11}$. Mais cette sublimation porte avec elle le risque de la sophistication et de l'artificialisme qui trahirait

\footnotetext{
${ }^{4}$ B.2, 32-33 : « Pan, en premier, à rassembler plusieurs chalumeaux avec de la cire, / Fonda l'art... » [Trad. F.C.]

${ }^{5}$ B.8, 25 : « Pan, qui, le premier, ne souffrit pas des roseaux sans art » [Trad. F.C.]

${ }^{6}$ [Pan]... Calamis agrestibus insonat ille / Barbaricoque Midan (aderat nam forte canenti) / Carmine delenit. (Mét. XI, 160-162) [Pan fait vibrer ses chalumeaux agrestes, / Et de son chant barbare il charme Midas (assis tout près du musicien)]. [Apollon] Artificis status ipse fuit. Tum stamina docto / Pollice sollicitat, quorum dulcedine captus / Pana iubet Tmolus citharae submittere cannas (Mét. XI, 169-171) [Le maintien même d'Apollon est celui d'un maître artiste. Alors il fait bouger les cordes / De son pouce savant, et séduit par leur suavité, / Tmolus ordonne à Pan que les roseaux se soumettent à la cithare.

${ }^{7}$ Mét. XI, 147 : Panaque montanis habitantem semper in antris...

${ }^{8}$ Cf NDIAYE Emilia, Un nom de l'étranger : Barbarus. Étude lexico-sémantique, en latin, des origines à Juvénal, P.U. de Lille (2001).

${ }^{9}$ Terme qui s'oppose, chez Homère déjà, à ßoukódos, Il. 17, 65, etc..., $c f$ BAILLY, p. 1331

${ }^{10}$ Dans les Bucoliques, Apollo est cité 6 fois, Phoebus 9 fois, Cynthius 1 fois, contre 7 fois pour Pan.

${ }^{11}$ Marchetta, A., Due studi sulle Bucoliche di Virgilio, I - L'incipit bucolico in Virgilio : ecl. 2, 1 ; II - La semantica di Formosus, Edizioni dell'Ateneo (1994)
} 
cette musique de l'Être dont les inspirés de Pan se font l'écho. Et du reste, la virtuosité n'est pas une garantie suffisante de séduction pour Virgile, comme le prouvent les échecs de Corydon (B.2) ou de Damon (B.8).

La poétique panique cherche donc, quant à elle, une autre beauté, un carmen deductum $^{12}$, résultant d'une « forme » qui soit en adéquation avec la vie, et que je qualifierais de jubilation. Pan, ne l'oublions pas, incarne les forces vives de la nature, la fécondité naissant de l'aridité. Individuellement, il est le prototype du berger $\left(\right.$ pas-tor $\left.^{13}\right)$, mais universellement, en vertu d'un jeu de mot que les Grecs s'autorisaient eux-mêmes, il est le dieu du Tout $(\Pi \alpha \alpha v)^{14}$. De par la nature polyphonique de son art, à l'image des sept ou neuf chalumeaux qui composent la syrinx, Pan exprime la faculté de réunir et de faire tenir ensemble la diversité de l'existence, de manière à atteindre une Unité de l'Être. La Beauté ne tient donc pas à la forme superfétatoire de l'art, mais à la rencontre d'une symbiose jubilatoire avec le monde.

Cette voluptueuse joie de vivre éclate avec générosité dans nombre de passages de Virgile. Célèbre est à ce titre l'enthousiasme avec lequel il clôt la Quatrième Bucolique : le poète, dans un élan prophétique, annonce le retour de l'âge d'or, et, oubliant toute modestie, rêve qu'il surpasserait, dans ces temps meilleurs, les plus grands poètes :

\section{Non me carminibus vincat nec Thracius Orpheus,}

Nec Linus mater quamvis atque huic pater adsit,

Orphei Calliopea, Lino formosus Apollo.

Pan etiam, Arcadia mecum si judice certet,

Pan etiam Arcadia dicat se judice victum ${ }^{15}$.

Orphée et Linos, deux poètes Enchanteurs, d'ascendance divine, appartiennent à ce temps improbable où la Parole poétique - le carmen, à la fois poème et «charme » - pouvait agir directement sur le monde. Fait inhabituel : Pan, qui est un dieu à part entière, est considéré lui aussi comme un poète Enchanteur et occupe même, dans cette hiérarchie, le rang le plus élevé. L'Arcadie représente l'instance suprême capable de juger (Arcadia iudice), dans la

\footnotetext{
${ }^{12}$ B.6, 5 : deductum dicere carmen, « dire un chant retréci », sous-entendu sans ampleur ni recherche de grandeur, promesse que tient cette bucolique, qui est une cosmogonie en miniature.

${ }^{13}$ Chantraine Pierre, Dictionnaire étymologique de la langue grecque. Histoire des mots, Paris, 1968-1980, montre la relation existant entre Pan et le radical pas- (en latin : pasci, pastus, pastor), dérivé du védique pati, « il protège », « il garde ».

${ }^{14}$ PlutaRQue, Sur la disparition des oracles ed. Flacelière, Dialogues Pythiques, Budé, 1974, p. 121-123 (Moralia 419 A 11-E 2); PLATON, Cratyle, 411a-421c

${ }^{15}$ B.4, v. 55-59 : «Non, Orphée le Thrace ne saurait me vaincre par ses chants, / Ni Linus, seraient-ils assistés l'un de sa mère, l'autre de son père, / Orphée de Calliope, Linus du splendide Apollon. / Pan aussi, avec l'Arcadie pour juge, s'il combattait contre moi, / Pan aussi, avec l'Arcadie pour juge, dirait qu'il est vaincu. »
} 
tradition des chants amébées, la qualité des poètes en lice : dans ce rêve, elle décrèterait la victoire de Virgile sur Pan.

On pourrait opérer certaines distinctions entre Orphée, Linos et $\operatorname{Pan}^{16}$, mais à la base, ils sont membres d'une même famille poétique, tout à la fois animiste et humaniste. Leur Parole, à l'aube du langage, «parle les choses » directement et trouve son harmonie avec elles. Les animaux et les forêts suivent Orphée (siluas sequentis - B.3, 46); Linos, l'inventeur du chant de lamentation (les $\Lambda^{\prime}$ ivoı, lui doivent leur nom), émeut même les rochers. Mais profondément ces deux poètes, et tous ceux qui traversent les Bucoliques (Silène, Daphnis, Tityre...) ne sont que des facettes multiples de Pan, qui crée entre eux le lien et la synthèse. Sans nul doute aussi, chacune des poètes apporte, dans sa différence, une nuance et une richesse supplémentaire : Linos exprime l'âme plaintive, Orphée la force agissante du chant qui meut les éléments physiques et métaphysiques ${ }^{17} \ldots$ On retrouve là les distinctions philosophiques d'une éthique (Linos) et d'une physique (Orphée) dont Pan serait le Logos central et constitutif.

La panodie, ou chant de Pan, vise donc un état originaire de la Parole (celui du logos organisateur mentionné par Héraclite) ou celle-ci fait un avec le monde, et non le langage constitué de signes (conception du logos diacritique pensé par Parménide ${ }^{18}$ ). De fait, la panodie virgilienne est plus qu'une pastorale, terme qui renvoie avant tout au genre, certes hybride, mais constitué. Néanmoins, cette Parole en interaction avec le monde, ne saurait être, tout au plus, dans la poésie de Virgile qu'une intention, et dans son écriture, la mimésis de cet état présumé premier. Elle nourrit, en tout cas, un horizon d'attente, ce que ressent à juste titre Yves Bonnefoy qui déclarait à propos de cette même Quatrième Bucolique :

(4) «Je découvris dans ces vers qu'une musique peut transgresser dans le mot ce qui fait notion de la chose, et permettre ainsi à ce qui est «vierge, vivace», comme dirait Mallarmé, d'apparaître à nos yeux dans l'intégrité d'avant le langage. ${ }^{19}$ »

Cette «musique » propre à la Parole poétique dépasse le réseau référentiel du langage normatif (où le mot renvoie à son objet, ou à son concept), et la fait accéder à un espace qui

\footnotetext{
${ }^{16}$ Notamment des distinctions génériques : cf. Collin F., Pan etiam Arcadia mecum si iudice certet, Virgile et l'esthétique arcadienne dans la poésie latine, Thèse Lettres Paris IV-Sorbonne (Avril 2005), p. 118 sqq. Orphée, par sa mère Calliope, désigne l'épopée, le grand genre du Destin collectif. Linus, le fils d'Apollon ou, selon les traditions, son rival poétique malheureux, incarne l'élégie, toute subjective. Epopée et élégie, les deux genres majeurs à Rome, se voient ainsi coiffer par ce genre nouveau que Virgile importe à Rome : la bucolique, dont l'essence est la panodie.

${ }^{17}$ Orphée est le poète qui meut les forêts et qui affronte aussi les puissances souterraines chez Virgile.

${ }^{18}$ HeRACLITE, frgts Diels-Kranz 1, 2, 50, 72, 108 pour la pensée du logos ; PARMENIDE, frgts Diels-Kranz 5 \& 7 pour le logos comme pensée critique.

${ }^{19}$ BonNEFOY Yves, Entretiens sur la poésie, «Quelques livres qui ont compté », p.339
} 
dénude la langue commune et la rende à un état primitif, plus «vierge, vivace ». Illusion de poète ? Je crois pourtant que Virgile a recherché cet état originaire de la parole, et qu'il s'est tourné, pour cette raison, vers les origines réputées les plus reculées, en son temps, de cette

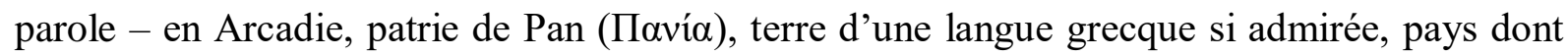
les habitants étaient, aux dires de Polybe, spontanément poètes et chanteurs ${ }^{20}$. L'admiration qu'il vouait à la poésie exerçait le même charme que son latin, aujourd'hui, sur nous :

(5) «Ces classes de latin, dont il fallait bien toutes les longues heures arides pour que finisse par apparaître, et peu à peu révèle sa qualité prosodique, sa beauté, un de ces vers de Virgile dont je crois essentiel qu'on l'ait découvert à quatorze ans, à quinze ans, quand l'imagination est encore assez enfante pour rêver d'un arrière monde, et accéder ainsi à la poésie: sans Virgile au Collège de Charleville, sans cette présence préservée là de la tradition pastorale, il n'y aurait pas eu Rimbaud, je n'en doute guère. ${ }^{21}$ »

On le voit, l'apprentissage d'une langue est long et « aride » (pas spécifiquement celui du latin !). Mais, dans la pratique de la langue d'un poète, l'aridité débouche sur une lumière, je dirais sur cette musique («qualité prosodique ») qui dévoile la partie «rêvée » forgeant la conscience de l'enfant et de l'artiste, que Bonnefoy nomme "arrière-monde » et qui correspond le mieux - nous tâcherons de le montrer - à ce que Virgile appellerait pour sa part «l'Arcadie ». Ce territoire poétique, nullement donné d'emblée, nourrit la conscience poétique et le dialogue continu entre les poètes : de Pan à Virgile, de Virgile à Rimbaud (dont la première production est celle de vers latins !), de Rimbaud à Char ou à Bonnefoy.

Rendre compte de la panodie arcadienne de Virgile revient à identifier une poétique mettant l'accent sur la musique et l'action directe de sa lyrique sur le monde. On ne pourra rendre compte ici que de quelques indices précis d'écriture...

De cette musique qui excède la langue, le jeu de la flûte est très symptomatique. Car que sera le «chant » de Pan si la bouche est occupée à souffler ? N'y aura-t-il aucun mot articulé, aucune conscience claire des mots ? Servius nous apprend que les Bucoliques étaient chantées et modulées sur scène à la manière, sans doute, d'un récitatif ou d'une mélopée. Mais il nous semble plus riche de prendre ici le carmen dans un sens métaphorique, c'est-à-dire de montrer

\footnotetext{
${ }^{20}$ Polybe, Histoires, IV, 5 : « L'exercice de la belle musique, qui n'est qu'utile aux autres hommes, est absolument nécessaire aux Arcadiens. »

${ }^{21}$ BONNEFOY Yves, « Remarques sur l'enseignement de la poésie au lycée », L'Esprit créateur n 106, 1996. Interrogé, en 1996, sur l'enseignement de la poésie au lycée, Yves Bonnefoy évoquait la relation qu'il avait eue, dans l'enfance, à la langue latine et à Virgile en particulier.
} 
que la langue latine, à partir des mots articulés par Virgile, entend restituer comme une musique plus unitaire de l'Être et du monde.

Ainsi les sonorités peuvent elles être étudiées pour leur effet matérialisant à l'intérieur du vers :

(6) hic uiridis ${ }^{\mathbf{T}}$ tenera ${ }^{\mathbf{P}}$ praetexit ${ }^{\mathbf{H}}$ harundine ripas

DDSD

$\begin{array}{lllllll}1 & 2 & 3 & 0 & 3 & 2\end{array}$

Mincius, eque sacra ${ }^{\mathbf{P}} \underline{\text { resonant examina quercu }}^{22}(\mathrm{~B} .7,12-13)$

DDDS 1

Les disjonctions donne à la disposition métrique un effet d'étirement du fleuve natal (grâce à l'enjambement hic... / Mincius), doublé d'un effet de circularité autour du verbe praetexit (rendue par la figure 1,2,3 - 3,2 / -1). Les sons accompagnent ce phénomène de douce lenteur: les dentales $[\mathrm{t}]$ et $[\mathrm{d}]$ reproduisent, comme une discrète basse continue, le débit alangui ; les vibrantes [r] laissent sourdre le bourdonnement actif des essaims ; quant aux nasales [n], ce sont elles qui fondent ses sons contraires et apportent une rondeur reposante (harundine - Mincius - examina) à l'ensemble.

Mais ailleurs, les sonorités sont de véritables architectures phoniques structurant un distique ou une strophe :

(7) Tityrus hinc aberat. ipsae te, Tityre, pinus,

DDSS

ipsi te fontes, ipsa haec arbusta vocabant ${ }^{23}$. (B1, 38-39)

SSSS

La répétition de ipse scandent la progression du distique jusqu'à son sommet clausulaire (vocabant) qui amplifie l'appel et l'impatience de la forêt attendant le retour de Tityre. Cette gradation des ipse, aux trois genres, suggère un sentiment unanime. L'accumulation syllabique de [ti] et de [tü] dissout le nom « Tityre » et le recompose comme paronomase dans le topiaire de la source (ipsi te fontes $=$ Tityre) ou efface son absence dans l'anagramme d'arbusta (inversion d'aberat). La musique, comme dans le mythe de Syrinx, comble une absence ou la décompense. Bien chanter, c'est donc se mettre en phase avec le monde.

${ }^{22}$ B.7, 12-13: « Ici il couvrit ses rives verdoyantes d'un tendre roseau / Mincio, et les essaims résonnent de son chêne sacré » [Trad. F.C.]

${ }^{23}$ B1, 38-39 : « Tityre était parti d'ici, et c'est toi, Tityre, que les pins eux-mêmes, / Toi que les sources elles-mêmes, toi que ces arbustes eux-mêmes appelaient... » [Trad. F.C.] 
La musique $\mathrm{a}$, plus encore, une véritable force de structuration de l'ensemble d'un poème. La critique s'est souvent interrogée pour déterminer les raisons objectives qui donnaient, dans le chant amébée de la Septième Bucolique, la victoire à Corydon sur Thyrsis. J.P. ChausserieLaprée a démontré que la structuration sonores des strophes étaient, sans équivoque, en faveur de Corydon qui présente un «nombre plus élevé de figures phoniques » (22 contre 12 chez Thyrsis), « une plus grande variété de types métriques » (6 contre 3), « une illustration phonique du rythme plus heureuse et plus soutenue $»(17 \text { contre } 4)^{24}$. La souplesse, la netteté et l'élégance font donc la supériorité musicale de Corydon.

L'espace lyrique où cette musique peut s' inscrire et agir, c'est la forêt (silva) : elle connaît une grande propension chez Virgile ${ }^{25}$, qui la revendique comme inhérente à l'art poétique de Pan que les poètes arcadiens doivent suivre :

\section{(8) Mecum una in silvis imitabere Pana canendo ${ }^{26}$ SSDD}

Cette «Muse silvestre» aux accents rudes ${ }^{27}$ agit selon plusieurs axes dont nous ne retiendrons ici, pour la démonstration, que deux actions, paradoxales et complémentaires. Il y a, d'une part, la fascination pour la petitesse (humilitas), de l'autre, la volonté de conciliation dans l'échange (alterna). En effet, la Muse silvestre est une muse de la célébration (laudatio) qui s'émerveille de l'être le plus humble, du végétal le plus discret,

(9) Munera sunt, lauri et suave rubens hyacinthus. ${ }^{28} D S D S$

L'attention portée au moindre détail, à la minutie de la forme, à la spécificité de la couleur - mais ailleurs à celle d'un parfum ou d'un son - pourrait dissoudre cette poétique dans une ekphrasis pointilleuse et lassante au final. Or, loin de se perdre dans le détail, cette poétique de l'ombre redonne de l'intérêt aux plus petits êtres, et hisse l'infime à un degré de grandeur inconnue ${ }^{29}$, action possible, parce qu'elle devient, dans la forêt, non seulement transitive, mais réciproque.

\footnotetext{
${ }^{24}$ ChAusSERIE-LAPREE J.P., « Les Constructions » de la Septième Bucolique », in J. COLLART, Varron, Grammaire et stylistique, Belles Lettres, Paris (1978). Voir aussi «Echos et résonances au début de la Dixième Bucolique », in Mélanges Pierre Boyancé, Collection de l'Ecole Française de Rome (1974).

${ }^{25}$ Les Bucoliques comptent à elles seules occurrences de silva contre deux seulement de ú $\lambda \propto$ chez Théocrite.

${ }^{26}$ B.2, 31 [Corydon s'adresse à Alexis] : « Avec moi dans les forêts tu imiteras Pan en chantant »

${ }^{27}$ B.1,2 : silvestrem Musam ; B.3, 84: rustica Musa(m). Silvestris prend aussi le sens voulu par Virgile de „sauvage“ par opposition à urbain et civilisé.

${ }^{28}$ B.3, 62 (chant de Ménalque) : « [Phébus toujours a chez moi /] Ses présents, les lauriers et l'hyacinthe doucement (délicatement) rouge. »

${ }^{29}$ Heuze Philippe, «L'esthétique du précis et l'esthétique du flou dans les Bucoliques », in R.E.L. nº84 (2006).
} 
Car les calami ont été inventés par Pan pour faire vibrer et chanter la forêt en retour. L'échange n'est donc pas seulement le répons autour d'un thème, tel que le pratique le chant amébée (B. 3 et 7), mais la possibilité d'entretisser, grâce au chant un dialogue avec la nature,

\section{(10) Non canimus surdis: respondent omnia silvae $\quad$ DSSS}

Ainsi s'orchestre une véritable réflexivité de l'homme avec l'Être, que les constructions chiasmiques, métriques $^{31}$, ou l'usage du préfixe [re-] mettent en scène, donnant là encore au vivant le plus petit sa dimension dans le $\operatorname{cosmos}^{32}$. Le carmen panodique enveloppe dans son incantation le monde telle une harmonie et une lumière, rapprochant d'un trait le sol et le ciel à la manière de Daphnis,

\section{(11) Daphnis ego in silvis hinc usque ad sidera notus ${ }^{33} \ldots \quad$ DSSS}

De même, le Silène (B.6) élève un chant cosmologique englobant, cette fois, le temps, et réunissant deux versants de la nature humaine, la fondatrice Pyrrha, et l'errante Pasiphae. Et Tityre (B.1), apprenant aux forêts à redire le nom d'Amaryllis, les fait résonner non seulement du nom de l'aimée, mais du sentiment même de l'amour (amare), traduit en musique. La panodie engage donc cet échange avec une unité retrouvée - celle d'une nature habitée, d'arbres et de divinités vivants - et restaure le lien de l'homme avec l'ici bas, avec « le vierge, le vivace. »

L'Arcadie tracée par la poétique virgilienne n'est en rien un lieu géographique, on le voit bien, mais une fiction poétique, un point de restauration de la Parole dans une immanence probable. Et ce qui importe à Virgile - comme un acte de foi - c'est de lui donner son ancrage dans la latinité. Mais en quoi cela n'est-il pas une illusion de plus ? En quoi le latin - le latin de Virgile - retrouverait-il une Parole originaire ?

Yves Bonnefoy, passé le cap de la fascination, ne peut se garder, dans L'Arrière-Pays, d'exprimer une réserve :

\footnotetext{
${ }^{30}$ B.10, 8 : « Nous ne chantons pas pour des sourds : en tout point les forêts nous répondent.»

${ }^{31}$ Par exemple, le chiasme strophique de B. 1, 1 : Tityre, tu patulae.../ B.1, 4 : Tu Tityre, lentus in umbra... Les dispositions métriques présentent également des enchaînements en réversibilité : DDSS / SSDD ; DDDS / SDDD [B. $1,1 \& 5]$, etc...

${ }^{32}$ COLlin F., ibid., p. 305 sqq pour les emplois de resonare, respondere, referre : formosam resonare doces (B.1, $5)$; pulsae referunt ad sidera valles (B.6, 84); diuom referatis ad auris (B.3, 73) ; Eurydicen toto referebant flumine ripae $(\mathrm{G} .4,527)$; vox reddita fertur ad auris $(\mathrm{E} .3,40)$; notas audire et reddere voces $(\mathrm{E} .6,689)$...

${ }^{33}$ B.5, 45 [épitaphe de Daphnis, après son apothéose] : «Je suis ce Daphnis, connu dans les forêts, d'ici jusqu'aux astres [berger d'un beau troupeau, moins beau que le berger] ».
} 
(12) «Virgile n'a pas parlé aussi profond, aussi autrement, que le latin semblait le permettre. Il s'est même leurré dans un ailleurs illusoire dans les montagnes de Grèce, avant de mourir dans ce «lieu où l'on passe », et où lui revint, Brindisi. [...] Ne faut-il pas remonter au-delà de ce moment de la poésie, simple vestige, première ligne des cimes, vers un état ancien de la langue, sinon même jusqu'aux dialectes qui ont précédé le latin... ${ }^{34}{ }$,

La critique est lourde et condamne la panodie virgilienne : Virgile, en maître pourtant reconnu de la langue, n'a pu néanmoins dépasser une raideur, une distance, qui l'eût rapproché d'un état assez ancien pour atteindre la Parole originaire. Il s'est « leurré » dans cet « ailleurs illusoire » que l'Arcadie offrait à sa conscience, au point de ne jamais la trouver, et de s'égarer en la cherchant. Un point biographique atteste que Virgile, au moment d'achever l'Enéide, aurait voulu se rendre en Grèce, et voir, peut-être, l'Arcadie de son rêve, mais, malade durant sa navigation, il n'aurait eu que le temps de revenir à Brindisi, le grand port de passage du sud de l'Italie. Il serait donc resté, pour parodier un titre de Bonnefoy, dans le leurre de ce seuil. Et Bonnefoy, suite à cet «échec » virgilien, se met à rêver à une langue plus profonde, à des « dialectes » plus anciens que le latin.

Pourtant, ce « leurre » est inhérent à toute poésie de l'origine, et Virgile a lui-même tenté de renouer avec les dialectes les plus archaïques dont se prévalait l'arcadisme en reliant l'héritage arcadien à la tradition oraculaire du faunisme latin. Varron nous indique, relativement à cette tradition ${ }^{35}$, que Faunus, Fauna, et les Faunes, divinités italiques, assimilées vers le $\mathrm{IV}^{\circ} \mathrm{S}$ av J.C. à Pan et Evandre, ont une fonction phatique forte, c'est-à-dire prophétique. Ces dieux premiers auraient exprimé eux-mêmes leurs oracles en vers saturnien ${ }^{36}$, un vers rituel proprement latin qui tomba en désuétude avec Ennius, au profit de l'hexamètre dactylique. Or Virgile a légitimé sa panodie, dans un souci tout la fois de poésie et de piété, en cherchant à reproduire, dans son propre hexamètre, la matérialité archaïsante du saturnien.

Les vers précédemment cités recoupent en effet certaines de ses caractéristiques : une colométrie binaire et marquée autour d'une coupe forte, à l'exemple des vers sur le Mincius étudié plus haut (citation 6) ; un poids des mots important, de préférence disyllabiques et

\footnotetext{
${ }^{34}$ Bonnefoy Yves, L'Arrière-pays, 1972, Skira, Champs Flammarion, p. 100.

${ }^{35}$ VARRON : De Lingua latina, 7, 36. «Les Faunes sont des divinités latines telles qu'il y a un dieu Faunus et une déesse Fauna; on rapporte qu'ils avaient coutume d'annoncer dans des lieux boisés l'avenir en vers saturniens et cette fonction phatique leur valut l'appellation de Faunes». Faunus serait donc dérivé du verbe fari, fando, fans, (« parler ») ; in-fans, «l'enfant juste né » est « celui qui ne parle pas encore ».

${ }^{36}$ DANGEL Jacqueline, «Faunes, Camènes, et Muses : le premier art poétique latin ?», Bollettino di Studi Latini, Anno XXVII, Fascicolo I, Naples (Juin 1997). DE GROOT (A.W.): «Le vers saturnien littéraire», R.E.L. XII (1934). PIGHI (G.B.): «Il verso saturnio», R.F.I.C. 35 (1957). COLE (Th.): «The saturnian verse», Yale Classical Studies, p.1-73 (1969). LuISELli (B.): Il verso saturnio, Rome (1967). BLÄNSDORF (J.): «Metrum und Stil als Indizien für vorliterarischen Gebrauch des Saturniers», Studien zur vorliterarischen Periode im frühen Rom, Tübingen (1989).
} 
trisyllabiques (citation 7) ; un rythme (numerus) garantissant au vers un relief très dessiné, selon des schémas métriques en alternance du type DDSS-, DSDS- ou DDDS-. Autant de moyens stylistiques par lesquels Virgile relate la panodie active des Faunes, comme une parole qui émane du monde et s'en fait l'écho, parce qu'ils en ont une vision totale :

(13) Tum vero in // numerum// Faunosque ferasque videres SDSDLudere, tum // rigidas // motare cacumina quercus ${ }^{37}$ DDSD-

Cette vision n'est pas une « connaissance », mais une révélation qui, à l'instar de la musique, provoque une transe extatique de participation dont la danse est l'expression : danse qui gagne la nature (quercus) et ses dieux (Faunos) dans une fusion rythmique unitaire.

Le très ancien collège des Frères Arvales ${ }^{38}$, parmi ses rites, en avait un qui consistait à danser solennellement (tripodare) sur des paroles en très vieux latin, formant des tripodies semblables à un premier membre de saturnien. Ces danses sacrées se nommaient tripudium, ce qui donne à penser que les tripodies jouaient un grand rôle dans la versification rituelle ${ }^{39}$. Elles étaient célébrées en mode «faunien», le plus ancien des modes connus ${ }^{40}$. C'est ce type de danses avec lequel Virgile renoue plusieurs fois dans son œuvre :

(14) Et vos, agrestum praesentia numina, Fauni, Ferte simul Faunique pedem Dryadesque puellae $e^{41}$

L'expression ferre pedem ne renvoie pas à un simple « déplacement » de ces divinités qui «s'avanceraient», car les Faunes exercent un talent qui leur est propre, en esquissant un pas de danse à côté des Dryades. Une telle danse traduit la force vitale dont ils sont porteurs, et cette intensité d'être (sens plus existentiel de praes-entia) rappelle qu'ils sont l'esprit des choses.

Cet état n'est pas sans rappeler celui de la voluptas lucrétienne, d'un amour ouranien qui posséderait tous les êtres et serait la nécessité du monde à qui sait la reconnaître, c'est-àdire par la poésie. C'est cette voluptas («plaisir de vivre, d'être ») qui s'empare de Pan et des Dryades et les fait participer à cette allégresse qui jaillit de la nature. C'est une force plus

\footnotetext{
${ }^{37}$ B.6, 27-30 : «De ci, tu aurais vraiment vu en rythme et les Faunes et les fauves / S'ébattre, de là les chênes rigides balancer leurs cimes. »

${ }^{38}$ Ovide, Fastes, IV, 629 sqq, éd. de Robert ShILling, Les Belles Lettres (1993) ; ShILling R., Rites, cultes, dieux de Rome, Paris (1979) ; ScHEID J., Le Collège des Frères Arvales, Rome (1990).

${ }^{39}$ Havet (L.) : Métrique grecque et latine, Paris (1930), p.217.

${ }^{40}$ PlACIDUS, $\$ 47$ : Faunorum modum, antiquissimorum modorum, quibus Faunum celebrabant...

${ }^{41}$ G.1,10-11 [Invocation liminaire des Géorgiques] : «Et vous, divinités favorables aux campagnards, Faunes, / Portez ici votre pas en même temps, Faunes et jeunes filles Dryades. »
} 
puissante que celle de l'amour (amor) qui risque sans cesse de devenir tragique et de détruire l'être.

\section{(15) Ergo alacris siluas et cetera rura uoluptas}

\section{Panaque pastoresque tenet Dryadasque puellas ${ }^{42}$}

Cette force énergétique du monde n'est donc un chant d'amour, mais une panodie, un amour du chant, si je puis me permettre ce jeu de mot. La langue n'est jamais qu'un leurre pour atteindre cette adéquation, mais, il faut la garder comme alliée, et non la rigidifier en image ou en concept. Virgile a, en ce sens, très sérieusement cru en une mission de la poésie sur le monde. Par-delà la fonction de poète vates, il s'agit plus profondément encore d'un optimisme tendu vers le renouveau du monde après le désastre des guerres civiles.

\section{LE PAYSAGE ARCADIEN}

Ayant défini la poétique arcadienne comme une panodie, attachons nous à définir son esthétique picturale, sa représentation du monde. Est-elle une idylle comme l'a affirmé Panofski, qui voit advenir chez Virgile la métamorphose de l'Arcadie indigente des Grecs, en Arcadie bienheureuse ? Le poète n'aurait donc que transposé en Arcadie le locus amoenus des Idylles de Théocrite $^{43}$ ? Ceci conduit une réflexion sur la technique paysagiste et l'adage horatien de l'ut pictura poesis (la poésie est-elle comme la peinture ?).

Le paysage occupe, dans l'Antiquité, une fonction, non pas réaliste, mais symbolique. Il est une succession de topö̈ (en latin, de loca), qui ont pour but de concourir à un effet du discours. Ainsi le locus amoenus (que l'on peut traduire par « lieu amène », « lieu plaisant ») est-il un lieu rhétorique, traité habituellement non pour lui-même, mais en contrepoint, notamment par les genres épique ou dramatique. C'est un paysage idéal ${ }^{44}$ qui, depuis Homère (les vergers d'Alkinoos, dans l'Odyssée) jusqu'aux bucoliques alexandrins, répond à une codification précise.

Horace, dans l'Art poétique, en donne l'esquisse, tout en défendant son usage dans le style épique :

\footnotetext{
${ }^{42}$ B.5,58-59 : «Ainsi une allègre jouissance possède les bois et toute la campagne / Ainsi que Pan, les bergers et les jeunes Dryades. » [Trad. E. de Saint-Denis]

${ }^{43}$ PANOFSKY (E.): L'Oeuvre d'art et ses significations, p. 282.

${ }^{44}$ CURTIUS, Ernst Robert : La littérature latine européenne et le Moyen-Âge latin (1947), cf « Le paysage idéal », chapitre tout à fait essentiel sur la question.
} 
et properantis aquae per amoenos ambitus agros ${ }^{45}$.

Le locus amoenus comprend donc l'espace d'une prairie (ager, prata, saltus), la proximité d'une eau courante (aqua, rivus, fons), l'ombrage d'un bois (lucus, nemus, silva, fagus, quercus), qui rappelle souvent la présence une divinité. Chez le Virgile de la Septième Bucolique, où les bergers poètes en présence sont dits Arcades (Arcadiens), on retrouve bien ces éléments :

(17) Muscosi fontes et somno mollior herba, et quae vos rara viridis tegit arbutus umbra ${ }^{46}$

L'ombre et la fraîcheur peuvent, à l'occasion, être suscitées par la présence d'une grotte (antrum, spelunca), élément plus montagnard, qui semble artificiellement rapporté, comme elle le serait pour la décoration d'un jardin. Ainsi, dans la Cinquième Bucolique, Ménalque et Mopsus choisissent-ils ce lieu dans le prélude à leur chant :

(18) sive antro potius succedimus. aspice, ut antrum silvestris raris sparsit labrusca racemis. ${ }^{47}$

Ce paysage a les caractéristiques d'une nature douce et bien ordonnancée, douceur qui, au dire de Varron, prédispose particulièrement à l'amour : ad se amanda adliciant ${ }^{48}$. On aura compris que cette esthétique répond à celle de l'idylle qui prend précisément pour sujet les amours des bergers dans un milieu agréable et champêtre. Or l'un des sens de « l'idylle », en

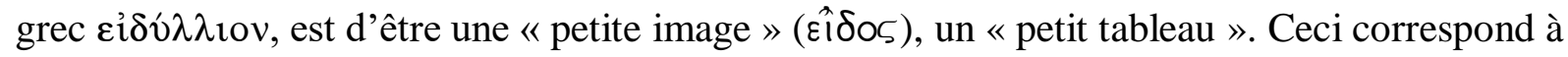
l'éthique du genre qui est celle de l'humilitas : comme il est impossible de représenter la nature dans sa totalité, l'esthétique bucolique se résout à la suggérer, par touches fragmentaires, et dans la miniaturisation de chacune de ses parties.

Mais cette esthétique risque aussi de se heurter, à la longue, à certaines limites qui serait de se réduire à n'être qu'une série de scènes obligées, dont, il est vrai, on retrouve quelques

\footnotetext{
${ }^{45}$ Ars poetica, v. 16-17 : «...un bois sacré et un autel de Diane / et les sinuosités d'un ruisseau rapide à travers une riante campagne. »

${ }^{46}$ B. VII, 45-46 (chant de Corydon) : «Sources moussues, gazon plus moelleux que le sommeil / Et toi, vert arbousier, qui répand sur vous son ombre clairsemée. » [Trad. Eugène de Saint-Denis]

${ }^{47}$ B. V, 45-46 (Mopsus à Ménalque) : « Soit que nous pénétrions plutôt dans la grotte. Vois comme cette grotte / A été tapissée, çà et là, de grappes clairsemées par la lambruche sauvage. » [Trad. Eugène de Saint-Denis]

${ }^{48}$ Ibid., XIV, 8, 33 : Amoena loca Varro dicta ait eo quod solum amorem praestant et ad se amanda adliciant.
} 
constantes : scène de la grotte, scène sous le pin, évocation du printemps, tableau des saisons. Le locus amoenus serait alors le lieu idéal et mièvre de la commensalité des dieux et des hommes rêvée sous l'Âge d'or, et l'idylle la représentation d'un monde lisse, d'un vert paradis des amours enfantines où l'on serait gavé de bonheur! C'est une illusion que reconnaît Bonnefoy :

(19) « «Simple», et la «terre », pour ne rien dire de cette idée d' « expérience », aujourd'hui révoquée comme subjective, ce sont là des notions on ne peut plus incertaines, je sais bien, et je n'ignore pas davantage que ces imprécisions, ces miroitements au loin créent le danger de nostalgies d'âge d'or, de rêveries pastorales, par quoi on perd le goût de la vérité. Mais un certain rapport à la vie, à l'être [...], cela a bien existé dans des sociétés dont une lumière nous vient encore, cela se présente encore... ${ }^{49} »$

Paradoxalement, les Bucoliques ne comporte pas une seule fois l'adjectif amoenus ! Si Virgile compose sur la base de topiaires, je pense que la portée de la technique paysagiste change radicalement, dans les Bucoliques et les recueils suivants.

Les Modernes croient avoir inventé la perspective et l'atmosphère, c'est-à-dire la manière dont un lieu, un paysage, une ambiance peuvent influencer l'esprit humain. Or, quand bien même la bucolique situerait son Arcadie dans un « ailleurs », et dans un cadre « hors du temps » (et pourtant pas anhistorique), cela ne légitime pas, à notre avis, une lecture allégorique de cette poésie.

Quelques grands savants se sont autorisé cette lecture : Léon Herrman voyait sous le masque de chaque berger des Bucoliques un correspondant réel, et la possibilité d'interpréter l'Arcadie virgilienne comme un cercle de poètes réunis en Cisalpine autour de la la personnalité d'Asinius Pollion. Pierre Grimal a reconnu pour sa part, dans le Daphnis de la Cinquième Bucolique, une allégorie de César, et la restauration par ce dernier de cultes agraires. La même attitude s'observe dans la postérité littéraire, si l'on se fie à l'émergence de la Pastorale en prose (ou roman pastoral) à la fin de la Renaissance, et par exemple, à l'Arcadia de Sannazar (1504) dont l'espace-temps est, selon Françoise Lavocat, je cite, « constitutif d'un dispositif herméneutique fondé sur l'allégorie. ${ }^{50}$

\footnotetext{
${ }^{49}$ BONNEFOY Yves, «Terre seconde », in Le Nuage rouge, Mercure de France, p. 347, 1976 [nous soulignons]

${ }^{50}$ LAVOCAT Françoise, «Topoï de la temporalité narrative dans la fiction en prose de l'Ancien régime : Le temps des bergers. Paradoxes et fictionnalité en Arcadie (XVI-XVIIes) » in Colloque La Pastorale, 16 juin 2003.
} 
Or, ce dispositif est toujours caution d'une clé d'interprétation, qui dénature la portée sémantique du texte littéral, et réduit son paysage à une image dont la troisième dimension, la plus signifiante, nous échappe. C'est une des raisons qui a marqué la lassitude pour le genre pastoral, condamné à n'être que l'imagerie kitsch d'un monde décalé.

Mieux qu'une allégorie, il me semble plus adapté de parler d'U-topie, en rendant à ce nom son sens «premier» (qui date, certes, de Thomas More, mais est fondé sur un hellénisme), à savoir de «Terre du Bien-être » (préfixe $\hat{\varepsilon} u$ ), ou mieux encore « Terre de Nulle Part » (préfixe oü).

La différence majeure tient au traitement des topiaires (des «lieux » marqueurs), et je tenterai d'en rendre compte par une comparaison de Théocrite et Virgile.

(20) $\underline{\text { B.9, 39-43 : }}$

«Huc ades, o Galatea: quis est nam ludus in undis? Hic uer purpureum, uarios hic flumina circum fundit humus flores; hic candida populus antro imminet et lentae texunt umbracula uites. Huc ades; insani feriant sine litora fluctus

"Viens ici, O Galatée: à quoi bon, en effet, perdre ton temps [dans les ondes? Ici c'est le printemps pourpré, ici le sol, au bord des fleuves, Epand ses fleurs diaprées, ici le peuplier blanc surplombe Ma grotte et les vignes souples tissent leurs ombrages. Viens ici ; laisse les vagues folles battre le rivage. »

\section{Id.11, 42-49 :}

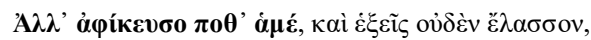

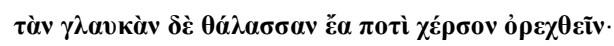

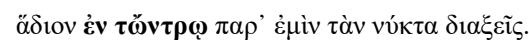

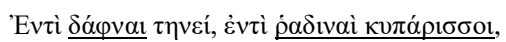

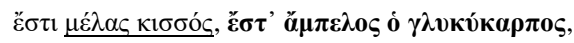

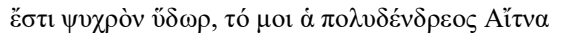

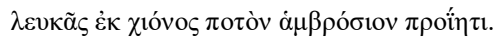

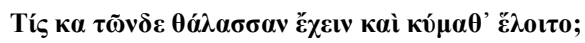

\author{
Allons viens à moi, et tu n'y perdras rien, \\ Laisse la mer verte mugir contre le rivage: \\ Tu seras mieux dans mon antre, près de moi, pour passer la nuit. \\ Il y a là des lauriers, il y a de sveltes cyprès, \\ Il y a du lierre noir, il y a une vigne aux doux fruits, \\ Il y a de l'eau fraîche que l'Etna couvert d'arbres, pour moi, \\ Extrait, divin breuvage, de la neige blanche.
}

Qui préfèrerait à cela habiter la mer et les flots ? ${ }^{51}$

L'imitation n'est pas, comme on serait tenté de le croire, une facilité, mais bien une contrainte. Le poète latin doit montrer qu'il reprend son modèle, et qu'il l'enrichit en l'adaptant. Il utilise à cette fin ce que j'appelle des « vers-cadres », c'est-à-dire des morceaux de vers traduits de son modèle (marqués en gras dans la citation 20) - dont il modifie, par des ajouts propres, la description, la perception, le sens.

Nous avons à faire, avec cet extrait de la Bucolique IX à l'un des premiers passages traduits par Virgile, qui suivait de près, à ce moment, la célèbre Onzième Idylle de Théocrite.

\footnotetext{
${ }^{51}$ Trad., peu modifiée, de Ph. Legrand, Les Belles Lettres
} 
Le Cyclope, traité de manière humoristique, tente de séduire, malgré sa laideur, la belle nymphe Galatée. Il l'invite à quitter la mer ondoyante pour le rejoindre dans sa caverne, espace terrestre tout à fait antithétique, en faveur duquel le Cyclope énumère maladroitement les qualités : lauriers, cyprès, lierre, doux fruits... C'est l'ombrage et la disposition d'avantages naturels qui plaident selon lui pour son mode de vie. Mais, allégoriquement, on comprend bien que ces modes de vie se dessinent sur un axe de parallèles inconciliables.

Virgile a repris le principe de l'opposition mer / terre (vers-cadre 1 et 5), mais a enchâssé le topiaire de la grotte (v. 4) dans une perspective plus large qui l'excède totalement. Avant de parler de l'antrum, il situe une saison (ver, le printemps), la proximité d'un fleuve (flumina), un peuplier (populus), toute la qualité terrestre d'un lieu amène et aimable. Car, détournant le caractère sombre des feuillages qui entourent la grotte (laurier, lierre, cyprès), il a opté pour la qualité sensible d'adjectifs colorés : «printemps pourpré » (purpureum), «fleurs diaprées » (uarios), peuplier d'un blanc éclatant (candidus). La terre donne l'image d'un séjour paisible, loin de cette folie (insani fluctus) des flots marins.

Cette terre virgilienne n'est pas allégorique, mais utopique. Où la situer ? Le paysage de Théocrite se verrait aisément dans les montagnes de Sicile : l'Etna est d'ailleurs nommé. Mais une grotte bordée d'un fleuve fertilisant? Les bords de Loire encore, du côté d'Amboise et de Saumur, mais pas la plaine du Pô. C'est donc un paysage de Nulle Part, non pas imaginaire, mais créé de pièces rapportées pour composer leur utopie.

Et cette utopie ne reproduit pas les topiaires idylliques d'une terre épargnée, elle rêve la possibilité d'une terre préservée qui déverserait sa douceur, son calme et sa beauté, qui donne aux deux poètes de la Cinquième Bucolique cette sensation physique d'être véritablement au monde, d'être « divin » :

(21) Tale tuum carmen nobis, divine poeta, quale sopor fessis in gramine, quale per aestum dulcis aquae saliente sitim restinguere rivo ${ }^{52}$

Nous voici rendus à une aporie. L'idylle nous donnait l'impression d'un monde réduit, mais réel et protégé. L'utopie nous semble composée de pièces rapportées, dessinant un «arrière monde » (expression de Bonnefoy) sans prise directe sur notre existence. Et c'est là qu'il faut sans doute se départir de dichotomies trop hâtives. Pourquoi l'utopie ne présenterait

\footnotetext{
${ }^{52}$ B.5, 45-47 [Ménalque s'adresse à Mopsus] : « Ton chant poétique est pour nous, divin poète, / Comme un somme sur le gazon pour l'être harassé, comme, en pleine chaleur, / Le plaisir d'étancher sa soif à l'eau délicieuse d'un ruisseau bondissant. » [Trad. E. de Saint-Denis légèrement modifiée]
} 
pas une vérité si elle est une traduction de la conscience poétique ? Plusieurs facteurs nous poussent ainsi à considérer l'Arcadie comme une utopie active, dans la poétique virgilienne, du fait que son idéalité n'est jamais virtuelle, mais bel et bien traversée par une limitation du concret, de la vie, de l'histoire, et du temps. Je donnerai trois exemples manifestes.

En premier lieu, l'histoire n'est absente d'aucun des trois recueils virgiliens. Dans les Bucoliques, Mélibée (B.1) ou Ménalque (B.9 - en qui l'on a voulu voir un «masque » de Virgile), sont les victimes des expropriations, et le poème accueille avec ferveur ces figures d'exil, rejetés de l'Arcadie possible. Je pourrais citer aussi la présence de personnages historiques, comme l'ami de Virgile, Gallus, disgracié par Auguste, et à qui le poète dédie courageusement la Dixième Bucolique. L'Arcadie n'est donc jamais donnée.

En second lieu, c'est un fait pictural, Virgile est sans doute l'inventeur de la couleur en poésie, et surtout du clair-obscur. Chez lui, l'ombre, et la nuit, n'offrent pas seulement leur fraîcheur ou leur apaisement, mais ajoutent un tremblement, une inquiétude, à l'intensité trop évidente du jour, en jetant un voile sur la pastorale idyllique. Sept des dix Bucoliques se terminent avec l'arrivée du Couchant (Vesper, Hesperus),

(22) Et sol crescentis decedens duplicat umbras. ${ }^{53}$

Aut, si nox pluuiam ne colligat ante ueremur ${ }^{54}$

Ite domum saturae, uenit Hesperus, ite, capellae. ${ }^{55}$

Les ombres, «dupliquées » par le soir, ne calment pas les angoisses de Corydon (B.2), mais le laissent en proie à son insoluble amour pour le bel Alexis (formosum Alexim, B.2, 2). Ménalque, exproprié de son domaine, est contraint de rejoindre la ville, et la nuit (nox ne veremur) que les orages rendent menaçante, le pousse à hâter le pas. Le tout dernier vers du recueil clôt l'univers arcadien (saturae capellae) et voit la nuit l'envelopper (venit Hesperus), en s'accordant à la tristesse de Gallus que cet univers n'a justement pu sauver de sa folie élégiaque.

Le vers est bien dans le fruit. L'Arcadie est menacée et par Eros et par Thanatos. Le Damon de la Huitième Bucolique se suicide par amour (quand cela se produit, chez Théocrite, c'est une hyperbole métaphorique, ou bien la vengeance d'une divinité, comme celle de Vénus qui condamne Adonis, réticent face à l'amour) ; Orphée, dans la Quatrième Géorgique, perd Eurydice, et meurt lacéré par les Ménades, épisode d'une fin qu'on ne trouve traitée de la sorte

\footnotetext{
${ }^{53}$ B.2, 65 : « Le soleil, à son déclin, double les ombres qui s’allongent ».

${ }^{54}$ B.9, 63: "Ou bien si nous craignons que la nuit n'assemble auparavant sa pluie sur nous... »

${ }^{55}$ B.10, 77: "Allez au logis, repues, voici le Couchant, allez, mes chèvres."
} 
que chez Virgile. Que dire encore de la mort de Pallas, le fils d'Evandre, ou de l'héroïne Camille, dans l'Enéide?

Cette découverte que la Mort et la Finitude traversent l'Arcadie a été mise en avant, au $\mathrm{XVII}^{\circ}$ S., par Nicolas Poussin, dans ses toiles admirables représentant Les Bergers d'Arcadie (1636), bergers réunis autour d'un sépulcre dans la campagne, et comprenant l'épitaphe Et in Arcadia ego... dont Erwin Panofski a remarquablement interprété le sens : «Et moi aussi, la Mort, j'habite en Arcadie» ${ }^{56}$. Gérard Genette a montré à son tour, dans un chapitre de Figures 1 , « le serpent dans la bergerie ${ }^{57}$, qu'Eros est ce serpent (celui qui pique Eurydice) dissimulé sous les idéaux de l'amour courtois, et que sa libido est menaçante pour l'univers où il s'inscrit. Genette reconnait du moins cette menace pour l'Astrée d'Honoré d'Urfé (1607-1619), mais non dans les formes antérieures et allégoriques de la pastorale en prose, l'Arcadie de Sannazar ou la Diana de Montemayor. Faut-il, du reste, expliquer l'intérêt moindre porté au genre pastoral, après le XVII ${ }^{\circ}$ S., par cette ruine de l'idylle et cette limitation de l'utopie ? Et s'il y a eu Chénier, Rousseau ou Hölderlin, l'Arcadie ne s'est-elle pas ruinée de l'intérieur en corrompant son idéal possible?

Tout au contraire, l'Arcadie n'est plus une imagerie. Elle a gagné, pour nous, avec Thanatos et Eros, la crédibilité d'un univers partagé entre aspirations spirituelles et suspens érotique, entre structure intemporelle du lieu et inscription de l'éphémère ("transiense" écrivait Panofsky en 1936, dans la première version de son article ${ }^{58}$ ), voire entre affirmation d'une vie "sans contraintes" et codification étroite de l'éthos de l'amour des bergers comme l'a souligné Norbert Elias ${ }^{59}$. Il n'y pas d'incohérence, dans l'ontologie moderne, marquée par l'épreuve du temps, pour qu'un lieu apparemment fictionnel ne soit l'expérience de quelque vérité, plus forte encore. J'en prends à témoin ce commentaire de Bonnefoy, concernant l'île de Capri - à qui il donne son nom ancien Capraia, « l'île des Chèvres », une île «pastorale » qui n'agit pas autrement sur lui qu'une Arcadie :

(23) « Capraia appartenait à l'Italie, rien ne la reliait à l'île où j'étais moi-même, on disait aussi qu'elle était presque déserte : tout se prêtait donc à ce que ce nom, qui la réduisait à

\footnotetext{
${ }^{56}$ PANOFSKY Erwin, “Et in Arcadia ego. Poussin et la tradition élégiaque ”, in L'Euvre d'art et ses significations. Essais sur les arts visuels, trad. de l'anglais par Marthe et Bernard Teyssèdre, Paris, Gallimard, " Bibliothèque des Sciences Humaines ", 1969 [1955].

${ }^{57}$ GenetTe Gérard, “Le serpent dans la bergerie ”, in Figures I, Paris, Editions du Seuil, 1966, p. 108-122.

${ }^{58}$ PANOFSKY Erwin, "Et in Arcadia ego". On the conception of Transience in Poussin and Watteau" : voir Louis Marin, "Poussin et Panofsky en Arcadie", article paru dans Erwin Panofsky. Cahiers pour un temps, Paris, centre G. Pompidou, 1983, repris in Sublime Poussin, Paris, Editions du Seuil, “L'ordre philosophique ”, 1995, p. 106124.

${ }^{59}$ ELIAS Norbert, "Curialisation et romantisme aristocratique ", La Société de cour [Die höfische Gesellschaft, 1969], traduit de l'allemand par Pierre Kamnitzer, Paris, Calmann-Lévy, 1974, rééd. Champs Flammarion, 1986.
} 
quelques bergers, à leur errance à jamais sur des tables rocheuses au ras du ciel dans le jasmin, l'asphodèle (quelques oliviers et caroubiers dans les creux), lui conférât une qualité d'archétype et en fit, pour ma pensée désirante, le vrai lieu. ${ }^{60}$ »

Ce lieu « vide », image même de la présence disparue, et de la permanence de l'Être grâce à « l'errance » des mêmes bergers, suscite, non l'image, mais la musique archétypale - de même que Pan était l'archétype des bergers - où pourra s'incarner tout « vrai lieu ». Ce vrai lieu ne se réfère ni à un lieu géographique (l'Arcadie du Péloponnèse), ni à un lieu allégorique (l'Arcadie idéalement promise), mais, dirait Plotin, à un «composé » (un koıvóv TI), irréel, mais non moins chargé de «présence » (praes-entia), et que nous allons tenter de préciser maintenant.

\section{EN MARGE DU REVE : UNE HYPOTYPOSE UTOPIQUE}

L'Arcadie a beau être, chez Virgile, un thème récurrent, elle demeure cependant plus impressive qu'objective, redevable, à distance, de ses influences littéraires, historiques et politiques, mais n'en retrouvant pas moins sa propre cohérence interne. L'Arcadie, qui incarne une patrie poétique, ne peut l'être qu'à la manière d'un rêve - mais ce rêve, cette utopie, sans être «illusion », garde en soi la synthèse de la présence. Aussi me semble-t-il plus adapté de parler à son sujet, non d'allégorie, mais d'hypotypose.

Pierre Fontanier définit en ces termes cette figure :

(24) « [L'hypotypose] peint les choses d'une manière si vive et si énergique, qu'elle les met en quelque sorte sous les yeux, et fait d'un récit ou d'une description, une image, un tableau, ou même une scène vivante ${ }^{61}$. »

Ce qui rend l'Arcadie virgilienne vivante, ce n'est pas d'abord la narration, ou la description, qui lui sont comme extérieures, mais bien son chant, son carmen panodique. L'Arcadie est le chant lui-même, saisissable le temps où se chant se produit, se terminant avec elle, ne laissant que sa trace onirique dans la conscience. Alors que l'on a souvent pensé la poésie antique comme récit ou description, je définis volontiers, quant à moi, l'Arcadie virgilienne comme «poésie pure », et comme l'essence même de la poétique de Virgile.

\footnotetext{
${ }^{60}$ BonNefOY Yves, L'Arrière-pays, 1972, Skira, Champs Flammarion, p. 13-14.

${ }^{61}$ FonTANIER Pierre, Les Figures du discours, Paris (1830; 1977), Ed. Genette, Flammarion, p.390.
} 
C'est la nature de cette hypotypose que nous voudrions préciser ici. Car, n'allons pas croire que tous les poèmes virgiliens seraient «arcadiens ", l'Arcadie n'est qu'un sommet de sa poésie, une Arcadia judice, «Arcadie juge » (citation 4). L'Arcadie suppose un horizon utopique, la mise en perspective d'une terre attentive et généreuse pour l'homme, et la tentative de produire les effets que son chant annonce : créer une symbiose harmonieuse avec une nature,

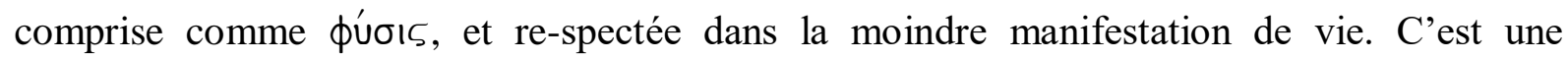
perspective qui dépasse les Bucoliques et s'observe bien dans les deux autres recueils virgiliens.

Dans ma thèse, $\mathrm{j}$ 'ai défini cette poétique arcadienne comme une technique utopisante. Le chant élabore sa construction, en s'appuyant sur une véritable mise en scène qui vaut comme une mise en condition : rencontre de bergers, situation prétendue anodine, rhétorique assez visible, clin d'œil aux modèles grecs. Mais le poème est bientôt dramatisé, et parvient, souvent en son milieu - à un point paroxystique ${ }^{62}$. L'échappatoire possible se dessine sous forme utopique, et l'Arcadie prend sa consistance comme cœur de ce poème. L'issue du poème, sa retombée, n'est pas modifiée d'ailleurs, par cette vision, comme nous l'avons dit précédemment : la fin n'est pas heureuse pour ces bergers - Tityre mis à part. Il y a parfois soulagement, non dans la satisfaction d'une passion, mais dans sa délivrance par la poésie, ce qui n'empêche pas quelque déception, ou même l'échec, pour Gallus, ou Damon.

La Première Bucolique, au premier abord, ne mentionne à aucun moment l'Arcadie. Son univers est pourtant implicitement présent : la pose de Tityre, qui rappelle son maître Pan adonné à sa sieste de midi ; le fagus, ce chêne-hêtre, constitutif de la vie primitive des Arcadiens qui se nourrissaient de glands. Tityre, autre Pan, autre chanteur arcadien, « apprend » aux forêts le nom d'Amaryllis, et jouit, au déclin de sa vie, de son domaine avec sa bien-aimée. Scène idyllique d'un égoïste qui n'intéresse pas Virgile. Car le drame vient de Mélibée, l'exproprié, obligé de s'exiler, et qui focalise en lui la nostalgie du perdant. Mais surgit alors, au milieu de la pièce, après un exposé sur la vie heureuse que mène Tityre, cette réplique (v. 46-58) où c'est Mélibée l'exilé, qui rend vivant, sous forme d'hypotypose, ce bonheur complet de Tityre, qui pourrait être le sien et celui de quiconque entend ce chant,

(25) Fortunate senex, hic inter flumina nota

et fontis sacros captabis opacum.

Hinc tibi, quae semper, vicino ab limite saepes

Hyblaeis apibus florem depasta salicti

\footnotetext{
${ }^{62}$ Voir aussi COLLIN Franck, «Mecum una in siluis imitabere Pana canendo, Le Chant arcadien dans la Deuxième Bucolique », Actes du XVème Colloque Guillaume Budé (2003).
} 
saepe levi somnum suadebit inire susurro. ${ }^{63}$

Ce rêve à voix haute de Mélibée rappellerait quelques vers-cadres de Théocrite (Id.7, v.135-143), et la vague description d'un Nymphée (fontis sacros). Mais l'image naïve est volontairement brouillée : dans le même champ, d'autres topiaires sont plus inattendus : un fleuve connu (flumina nota - le Mincius ?), une haie (saepes) délimitant une propriété, un saule (salicti - arbre convenant mieux aux rives d'un fleuve que d'une fontaine); des abeilles de l'Hybla (une montagne de Sicile ! Tandis que le père de Virgile, dit Donat, possédait des ruches et pratiquait l'apiculture). Est-on en Sicile ? A Mantoue ? A Naples ? Non, en Arcadie : il est inutile, à partir de ces brouillages intentionnels, de chercher une autre identification. Les topiaires composites ne reproduisent pas un lieu en particulier, ils esquissent l'U-topie, «Terre de Nulle Part », où chacun peut retrouver d'aventure des bribes de sa propre expérience. Ce qui importe à l'Arcadien, c'est que sa relation à l'Être (« nature») engendre cette intelligence réciproque et bienveillante des choses et forme une union concertante dont résulte le bien-être.

De cette perception, Bonnefoy rend compte, en évoquant l'Arcadie d'un autre Mélibée, celle de Werther chez Goethe :

(26) Tout poème [...] recèle en sa profondeur un récit, une fiction, aussi peu complexes soient-ils parfois: car la langue qui structure son univers ne peut que cristalliser en apparence d'objets ou d'êtres qui entretiennent entre elles des relations signifiantes, où paraît la loi même qui a présidé à la création. Or, cette fiction devrait, comme quintessence d'un rêve, en exprimer le bonheur, et aussi bien le fait-elle [...] même Werther est une Arcadie ${ }^{64}$.

Bonnefoy énonce que, par-delà la langue, avec son réseau sémantique, et ses images vite devenues clichés, surgit cette « loi » présidant à la création, je dirais : cette poétique qui repose sur l'expérience la plus profonde qu'un créateur fait des choses, et qui, même désespérée - celle de Mélibée l'est, au même titre que celle de Werther - reste « bonheur » ou « lucidité » sur le vrai. L'Arcadie dessine ainsi cet au-delà du chant qui est le vrai lieu de la poésie.

Le paradoxe du lyrisme arcadien est là : son utopie semble si évidente de netteté qu'elle en paraît « vraie ». S'appuyant sur des éléments concrets, elle l'est ; mais en même temps, elle n'est pas permanente, elle subsiste à l'état de souhait, ce que montre encore la prise en charge du discours par Mélibée. Pour nous modernes, l'utopie est balayée comme simplement illusoire et porteuse d'idéologie trompeuse. Les Anciens, poètes ou philosophes, s'en servent comme

${ }^{63}$ B.1, 51-55 : Heureux vieillard ! Ici parmi ces fleuves familiers / Et les fontaines sacrées, tu trouveras la fraîcheur de l'ombre. / De-ci, te délimitant du voisin, la haie, comme toujours, / Dont les abeilles de l'Hybla butinent la fleur du saule, / Souvent t'inclinera de son léger bruissement vers le sommeil.

${ }^{64}$ BonNefoy Yves, La présence et l'image, Mercure de France, 1983 (Collège de France, 1982), p. 35. 
hypothèse de pensée. Il ne s'agit pas, pour Virgile, de « séjourner » dans l'utopie, mais de projeter sous nos yeux ses bienfaits de manière si tangible.

Qu'est-ce qui garantit au poète la validité de son Arcadie ? La réussite de l'œuvre ? Le fait, disais-je au début, que toute la critique voyait l'Arcadie partout, sans la cerner dans sa cohérence, d'un recueil à l'autre ? La «beauté » tout simplement, elle que Rimbaud pensait devoir injurier ? Oui, la beauté qui semble innée et si peu donnée à quiconque veut la saisir :

(27) « Rien n'est immédiat, rien n'existe même, tout se gagne au cours d'une vie, et d'abord la beauté du lieu qu'elle assume. ${ }^{65}$ »

La beauté, je la situerai pour ma part, chez Virgile, aux frontières de l'existence : celles du Désir et de la Finitude ; d'une part l'Enfance, comme appétit de vivre, d'autre part la Mort comme nécessaire destruction. Or la qualité de l'Arcadie tient à la sensibilité que Virgile s'est forgée, sur sa terre natale de Mantoue, en Cisalpine. Les flumina nota (citation 26), le uiridis Mincio (citation 6), la plaine où il promet d'élever un temple à Octave (G.3, 13-16), la grande Etrurie de l'Enéide (chant VIII-X), le domaine paternel décrit par Lycidas :

$$
\text { Qua se subducere colles }
$$

incipiunt mollique ingum demittere clivo, usque ad aquam et veteres iam fracta cacumina fagos. ${ }^{66}$

sont les lieux d'une Arcadie oedipienne que Bonnefoy connaît avec le même charme bucolique dans le Lot de son enfance :

(29) [A propos de Toirac ] «C'était cette vallée, cette rivière là-bas, ces collines, le pays de l'intemporel, la terre déjà un rêve où perpétuer la sécurité des années qui ne savent rien de la mort. [...] Pays, par conséquent, d'une conscience qui peut appréhender l'univers (d'une façon naïve, qu'il faudra vite qu'on réprime) non dans le heurt déjà des existences finies, mais dans la musique des essences. ${ }^{67}$ »

La Transpadane pour Virgile, le Lot pour Bonnefoy, ont été ce socle qui soude la sensibilité et marque la conscience, en investissant dans la terre une vérité ontologique et non

\footnotetext{
${ }^{65}$ BonNEFOY Yves, L'Arrière-pays, 1972, Skira, Champs Flammarion, p. 71

66 B.IX, 7-9, Lycidas à Moeris : « depuis l'endroit où les coteaux / commencent à s'abaisser et à descendre en pente douce / jusqu'à l'eau et aux vieux hêtres à la cime déjà brisées. »

${ }^{67}$ Bonnefoy Yves, L'Arrière-pays, 1972, Skira, Champs Flammarion, p. 94
} 
telle ou telle imagination d'un arrière-monde fictif. La psychologie ou l'autobiographie importent peu ici, ce qui importe c'est la perception des correspondances restituant la qualité de l'affectivité première au monde, qu'on l'appelle «l'Un» (Plotin), « l'Ouvert» (Rilke) ou «l'Être » (Heidegger). C'est une entrappartenance que le nom, dans sa délimitation stricte et isolante, ne peut saisir, mais que le poète recompose comme terre seconde par le creuset de sa sensibilité.

L'Arcadie n'est donc pas la Transpadane, même si affleurent à sa surface des lieux enfouis, des souvenirs passés, des figures absentées, des voix évanescentes, par touches insaisissables, anciennement vécues : les flumina nota, le uiridis Mincius, le florem bene olentis anethi (B.2, 48), le levi susurro des abeilles (citation 25). Ce sont les signes minuscules, les «poussières d'or ${ }^{68}$ qu'il faut rendre au bonheur de l'instant où ils furent cueillis et qui constituent le seul vrai lieu.

(30) «La terre est, le mot présence a un sens. Et le rêve est, lui aussi, [...] ce sera lui le creuset où l'arrière-pays, s'étant dissipé, se referme, où l'ici vacant cristallise. Et où quelques mots pour finir brilleront peut-être, qui, bien que simples et transparents comme le rien du langage, seront pourtant tout, et réels. ${ }^{69}{ }^{»}$

Dès lors, dans le sens de la panodie décrite auparavant, s'instaure une tension entre le «Je » biographique et le «Je » universel, permettant au «Je » lyrique, à cette figure de Pan qu'incarne tout sujet lyrique, de s'inscrire dans le Tout, de s'emparer des quelques mots qui lui font saisir cette unité. Ce qu'Yves Bonnefoy traduit très concrètement dans son poème en prose, justement intitulé L'Origine de la parole, où l'on ne trouve rien de théorique que l'expérience de cette parole dans le vécu :

(31) «Et je comprenais que l'été est le langage. Que les mots naissent de l'été comme laisse un serpent derrière soi, à la mue, sa fragile enveloppe transparente. Que ce n'avait pu être qu'au sud, dans les miroitements du sel sur le roc - et ces buissons ardents ! et ces grands orages qui errent... - qu'on avait inventé les mots, et par eux l'absence ; qu'on avait rêvé la parole. ${ }^{70}{ }{ }^{\prime}$

Et l'on songe au prélude de la Deuxième Bucolique : à l'heure la plus chaude de midi, quand les moissonneurs s'interrompent dans leur tâche, pour déjeuner, Corydon se lance dans

\footnotetext{
${ }^{68}$ Bonnefoy Yves, Les Planches courbes, 2005, Poésie Gallimard, p. 108

${ }^{69}$ BonNEFOY Yves, L'Arrière-pays, 1972, Skira, Champs Flammarion, p. 136

${ }^{70}$ BonNEFoY Yves, «L'Origine de la parole », poème en prose, 2ème $\S$, in Récits en rêve, Mercure de France, 1987. Voir aussi « Le Myrte», in Pierre écrite (1965).
} 
une chasse sans espoir, pour rechercher un absent, Alexis, qu'il aime, mais que le maître a conduit avec lui à la ville,

(32) at mecum raucis, tua dum uestigia lustro, $\quad$ SSDS sole sub ardenti resonant arbusta cicadis ${ }^{71}$ SSDS

Le soleil tape fort, et les cigales, dans leur chant rauque en écho (resonant), amplifié par la disjonction, avertissent Corydon qu'il dévie de la raison, qu'il verse dans une folie passionnelle (dementia). Ces « buissons ardents » vont bientôt lui tendre la vision de la Naïade, de ses fruits et de ses fleurs (B.2, 45-55), et leur «miroitements » lui restituer le sens poétique, car le chant est nécessairement le reflet d'une absence. Et l'on y ajouterait la « mue » d'Orphée (G. IV, 315-558), qui, parti chercher Eurydice, morte de la morsure d'un serpent, n'en vient lui aussi qu'à troquer son apparence humaine pour n'être que la voix de l'Absence, ou du caché, si l'on préfère.

Or, disions-nous, l'autre dimension de ce lieu, c'est le poids de la finitude, le regard de la Mort. Cette relation à thanatos et à la fragilité ${ }^{72}$, très marquée chez Virgile comme chez Bonnefoy, n'est sans doute pour eux qu'une manière de rendre plus vivace, plus pure cette vie que nous traversons comme l'éclair.

Ainsi le lieu eschatologique que nous montre le chant VI de l'Enéide ne m'apparaît pas comme l'au-delà de la vie mais bien comme un ici dont la mort transfigure la présence, en nous en montrant les délices et la splendeur. Caractéristique est donc cette hypotypose des Champs Elysées, ce lieu situé à l'extrémité des Enfers, où vont les plus méritants :

\section{(33) Deuenere locos laetos et amoena uirecta}

fortunatorum nemorum sedesque beatas.

largior hic campos aether et lumine uestit

purpureo, solemque suum, sua sidera norunt. ${ }^{73}$

\footnotetext{
${ }^{71}$ B. 2, 12-13 : « Mais avec moi résonnent, rauques, pendant que je te suis à la trace, / Sous le soleil ardent, les buissons emplis de cigales. » [Trad. FC]

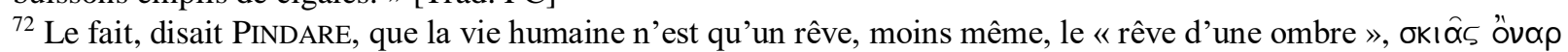

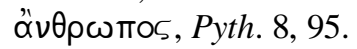

${ }^{73}$ E. VI, 638-641 [La Sibylle de Cumes et Enée] : «Ils descendirent vers des lieux gais et des pâturages amènes /, séjours bienheureux des bois des Fortunés. / En ce lieu, un éther plus généreux revêt les plaines d'une lumière / pourpre, et l'on connaît son propre soleil et ses propres astres. [suite v. 642-644: Les uns exercent leurs membres sur des palestres herbeuses, / s'affrontent dans des jeux et luttent sur le sable fauve ; / d'autres, battant du pied, rythment des choeurs et chantent des poèmes.] »
} 
La tradition grecque s'étend fort peu sur la partie heureuse des Enfers, et lui préfère ce lieu terrestre des Îles des Fortunés, si l'on en croit Homère et Pindare ${ }^{74}$. De la fusion de ces deux conceptions, d'inspiration orphique, se construit l'Elysée virgilien : ce qui d'abord en jaillit, c'est la lumière bienfaisante (lumine purpureo, solem suum) qui provient d'un « éther » second (aether, sidera) qui suffit à « nourrir » les bienheureux (fortunatorum); puis, élément de tradition plus latine et dans lequel on reconnaît l'influence de Lucrèce ${ }^{75}$, la présence de «lieux » (locos) que vient vêtir (vestit) et ourler une lumière exceptionnelle (purpureo).

Pourtant, toute comparaison faite avec Lucrèce, on retrouve là bien plus que la beauté d'un jardin : les contrées y semblent plus vastes et libres : locos, uirecta, sedes, campos. On y retrouve aussi tous les topiaires plaisants : l'adjectif amoenus, bien présent cette fois ; les virecta, dérivés du verbe virere; la joie (laetos, beatas) ; la lumière ; le lieu d'élection des Bienheureux (fortunatorum). Aussi cette description, de tradition orphique, possède toutes les marques de l'arcadisme virgilien: l'aspiration à la paix, l'oubli de la guerre ${ }^{76}$, le choix d'activités essentielles, comme l'exercice physique de la lutte ludique, et surtout celles de la poésie et de la danse.

Cette Arcadie est désormais métaphysique : elle donne visage à une vie de l'au-delà, une vie qui finalement n'est pas autre que celle que nous pourrions mener ici-bas si nous pouvions y tendre durant notre séjour terrestre, grâce à la poésie. Car, parmi les représentants de cette sagesse métaphysique, il n'y a ni divinité, ni philosophe ni politique, mais deux poètes : Orphée et Musée. L'un et l'autre (Musée fut, selon le mythe, disciple d'Orphée) passent, comme les Arcadiens, pour des inventeurs d'une civilisation fondée sur la poésie. Mais tandis que Pan est connoté du côté des forces vitales et des cycles de renaissance, Orphée a traversé la mort, et l'a vaincue, non charnellement, mais par cette Parole qui continue à errer par sa bouche, et demeure profondément énigme.

L'espace des Bienheureux apparaît ainsi comme la réalisation de l'Arcadie, un rêve qui trouverait dans l'absence sa vérité. Les êtres n'habitent plus de maison, comme les humains, mais seulement une vaste campagne ouverte, qui est extraordinairement terrestre, de par la fusion totale qui existe avec l'environnement :

(37) Nulli certa domus; lucis habitamus opacis,

\footnotetext{
${ }^{74}$ Homère évoque de façon sommaire les Champs Elysées (Od., 4, 561-568 ; 6, 42-44). Quant à Pindare, il consacre une longue description aux Îles des Bienheureux comme lieu eschatologique (Olymp., 7, 93-159).

${ }^{75}$ De Nat. Rerum I, 1-43.

76 E. VI, 652-653 : Stant terra defixae hastae passimque soluti / per campum pascuntur equi, « les javelines se dressent, fichées en terre, et les chevaux sans licou / paissent dispersés dans la plaine. »
} 
riparumque toros et prata recentia riuis

incolimus. ${ }^{77}$

Anchise lui-même, installé dans un vallon verdoyant, contemple ses descendants qui vont s'incarner $^{78}$. A côté de l'imagerie traditionnelle des Enfers, ce paysage devient le lieu de fixation d'une piété suprasensible, une Arcadie recomposée qui garde dans son au-delà sensible, la confiance dans le sens de la vie et de l'homme, et non la projection d'un idéal illusoire.

Ainsi l'Arcadie, comme vrai lieu, prend sa consistance de ce regard jeté par la Mort, capable de bouleverser la réalité et forçant la conscience à faire l'expérience d'une permanence toute relative à l'intérieur de l'Être, et qui tient à si peu de signes que la poésie a pour tâche de noter sans cesse. Claude Esteban, un autre lecteur aiguisé de Virgile, affirmait que le poète mantouan était, à ses yeux, le premier, à traduire dans sa poésie une composante empirique, une incertitude temporelle, qui faisait défaut aux grandes œuvres qui l'avaient précédé,

(35) «Les seuls dieux, dit-il, que Virgile révère - par-delà les fables d'une mythologie se sont les morts $^{79}$. »

Il s'appuie, pour sa part, sur un extrait des Géorgiques qui imagine les découvertes archéologiques des temps futurs et la surprise de trouver en terre les vestiges d'armes ayant appartenu à des êtres plus vigoureux ${ }^{80}$. Il y a, dans ce passage, l'éveil d'un savoir subjectif et la conscience d'une finitude qui traduisent la naissance du lieu poétique, lieu en réserve et en mutation, qui, chez Virgile, s'appelle Arcadie.

La confrontation de Virgile et d'Yves Bonnefoy nous a permis, par-delà leurs différences d'esthétique, de donner tout son poids à la fonction poétique, comme visée de la Parole, et quête du sens. Il nous est ainsi apparu que l'Arcadie virgilienne, malgré sa fragmentation et son

\footnotetext{
77 E. VI, 673-678 [Musée répond à la Sibylle] : « Personne n'a de demeure assurée ; nous habitons en des bois sombres, nous habitons les lits des rives et les prés frais des rivières. »

${ }^{78}$ E. VI, 679-683 : At pater Anchises penitus conualle uirenti / inclusas animas superumque ad lumen ituras / lustrabat: «De son côté, le vénérable Anchise, au fond d'une vallée verdoyante, passait en revue les âmes prisonnières destinées à rejoindre la lumière d'en haut... »

${ }^{79}$ EsTEBAN Claude, Un Lieu hors de tout lieu, «Le temps, la terre, le poème », Galilée, p. 30, 1979.

${ }^{80}$ G.1, 493-495 : Et tempus ueniet cum finibus illis / Agricola incuruo terram molitus aratro / Exesa inueniet scabra robigine pila...Et sans doute viendra le temps, là-bas, dans ces contrées / Où le laboureur, retournant la terre avec la charrue courbe, / Trouvera des javelots rongés par une rouille lépreuse / Et de sa houe pesante butera sur des casques vides / Et il s'étonnera de voir parmi les tombes ouvertes des ossements géants...»
} 
éparpillement dans les trois recueils, structurait, à la manière d'un arrière-pays, la conscience poétique du mantouan.

Virgile a conçu son chant, le carmen, sur le modèle de Pan et des poètes Enchanteurs, c'est-à-dire comme une panodie, mimésis d'une Parole originaire, englobant le monde dans sa vérité la plus humble, et capable d'agir sur lui en recomposant l'harmonie entre ses parties. Il a cru profondément en une mission de ce chant capable de changer son époque, tandis que l'historiographie utilisait de son côté l'Arcadie comme outil de propagande politique.

L'Arcadie n'en est pas moins un lieu, un lieu qui n'est ni géographique, ni allégorique, mais un «vrai lieu» dans l'acception que lui donne Yves Bonnefoy. Ce lieu conditionne la poétique de Virgile comme son point de tension et sa part rêvée, à la fois comme creuset de son expérience au monde et comme un idéal possible, mais insituable. C'est à ce titre que le terme d'utopie, au sens positif, nous parait le mieux s'appliquer à l'Arcadie virgilienne.

L'Arcadie est, enfin, un univers en construction, à travers lequel Virgile se livre à une recherche du sens, orienté à la fois du côté oedipien de l'enfance, et du côté de la contingence et de la destruction, dimension tout à fait novatrice dans l'Antiquité. Ce sont autant de raisons qui ont conféré à son Arcadie cet intérêt dans la littérature universelle, de lui jusqu'à Rimbaud, de Rimbaud jusqu'à Bonnefoy.

Franck COLLIN 